\title{
Complex analysis of polymer wastes in coal en- richment for the purpose of their utilization
}

\author{
Sergey Evmenov ${ }^{1}$, Galina Evmenova ${ }^{2,1}$, and Vladimir Tretyakov ${ }^{1}$ \\ ${ }^{1}$ T.F. Gorbachev Kuzbass State Technical University, Department of Coal Chemistry, Plastics \\ Materials and Environmental Engineering, \\ ${ }^{2}$ T.F. Gorbachev Kuzbass State Technical University, Department of Mineral Processing, Bld. 28, \\ Vesennyaya Str., 650000 Kemerovo, Russian Federation
}

\begin{abstract}
At 'wet' methods of minerals processing in order to dewater flotation concentrates, flotation waste and low-ash coal slurry, highly efficient equipment is used such as disk vacuum filters, belt filter presses, etc. On the filter surfaces of these machines, a filter cloth made of polycaproamide fiber is attached or put on, which eventually goes out-of use for various reasons and is transported to dumps (landfills). The paper presents a complex of physical and chemical analyses to evaluate influence of operating conditions of the filter cloth on the thermal and technological properties of the polymer. The results of the study showed a possibility of re-processing (recycling) of the out-of use filter material from vacuum filters in order to obtain marketable recycled polycaproamide.
\end{abstract}

\section{Introduction}

Re-processing (recycling) of out-of use plastic products is an important environmental problem for most industries, since these materials do not decompose under natural conditions for a long time.

One of the relatively expensive polymer materials with a number of valuable chemical, physical and mechanical properties, that is widely used in the mining industry and coal enrichment, is polycaproamide (polyamide PA-6). It is used for making sliding bearings, hoppers, various gaskets, seals in pneumatic systems, separators, drums for grinding rock, conveyor rollers, scrapers and etc. Polycaproamide fibers are extremely strong and take the first place in terms of production scale among other polymers. One of the ways of their use, in particular, in minerals processing is production of cloth (screens) for filtering surfaces of the disk vacuum filters and belt filter-presses at dewatering of flotation concentrates, lowash slurry and flotation waste. After the use, a cloth is removed to dumps (landfills), herewith the criterion of suitability is not the properties of the polymer, but the violation of the integrity of the screen caused by abnormal mechanical impact. Based on the results of a complex of physical and chemical analyses, this work herein studied, possible influence of the screens operating conditions on the polymer thermal and technological properties, in order to evaluate a possibility of obtaining marketable recycled polycaproamide, the raw material for which could be out-of use filter cloth from vacuum filters. 


\section{Materials and Methods}

For comparative analysis, samples of unused (new) filter cloth (sample \#1), cloth after its use where a content of coal particles in the cloth openings reached $20 \%$ by weight (sample \#2), and filter cloth washed from coal (sample \#3) were taken. Screen samples were crushed by cutting and studied by thermal and gravimetric analysis and infra-red spectroscopy. Density, thermal stability and moisture of samples were determined, and rheological properties were evaluated by a value of the Melt Flow Index MFI (an equivalent term is 'melt index' used in English literature). Properties of the recycled polycaproamide obtained by grinding and subsequent extrusion granulation of screen contaminated with coal particles were also studied (sample \#4).

To select processing methods and operating modes, as well as ways of the waste use, it is important to study thermal behavior of the out-of-use products and rececled materials obtained on their basis [1]. Therefore, in this paper, a complex thermal study of the above samples was performed by derivatography method that included thermal gravitational (TG), differential thermal gravitational (DTG) and differential thermal (DTA) analyses. The results were obtained using a Q1500D derivatograph (Hungary), which design was updated by adding ICP I-7018P module (ICP DAS, Taiwan) for analog-to-digital conversion of the T, TG, DTA and DTG signals using SIMP Light SCADA software, Russia) and Origin Pro software package (OriginLab, USA).

The TG and DTG curves obtained during the analysis are similar and only slightly differ in temperature limits that is shown in Table 1 . A value $T_{1}$ is temperature at which the thermal gravitational curve deviates from zero horizontal line and characterizes the beginning of the process of substance weight changing. At the temperature $T_{2}$, total decrease in the substance weight reaches its maximum value that corresponds to completion of the thermal decomposition reaction. The DTA curves having two pronounced peaks: heatabsorbing and exothermic, are also very similar for all samples and characterize thermal effects occurring in the tested samples. The heat-absorbing peak illustrates a process of polymer melting and is characterized by the melting temperature $\mathrm{T}_{\text {melting, }}$, its value for all samples is shown in Table 1, and is in the range of $205-212^{\circ} \mathrm{C}$ (Centigrade). For marketable primary polyamide PA- 6 melting temperature is regulated by $215^{\circ} \mathrm{C}$ value. An intense exothermic peak characterizes combustion of carbon residue at temperatures range $523-534^{\circ} \mathrm{C}$. For the recycled granulated polycaproamide this temperature is slightly higher and is $554^{\circ} \mathrm{C}$. The intermediate not pronounced peaks are referred to different stages of the polymer thermal decomposition.

Analyzing the obtained results, we can affirm that thermal properties of the samples are rather similar, and the process of the screen use did not lead to any serious changes in them. The IR spectrum of the sample 2 showed complete identity with the IR spectrum of the unused screen (sample 1) and literature data [2-4], as well as the presence of absorption bands typical for polycaproamide $\left(-\mathrm{NH}, 3300 \mathrm{c}^{-1} ;-\mathrm{CH}_{2}, 2936\right.$ and $2943 \mathrm{c}^{-1}$; $-\mathrm{C}=\mathrm{O} .579 \mathrm{c}^{-}$ $\left.{ }^{1}\right)$ and absence of the new one.

Table 1. Results of derivatographic analysis of the samples under study

\begin{tabular}{|c|c|c|c|c|c|}
\hline $\begin{array}{c}\text { Samples of } \\
\text { the mate- } \\
\text { rial under } \\
\text { study }\end{array}$ & $\begin{array}{c}\text { The } \\
\text { DTA } \\
\text { curve }\end{array}$ & \multicolumn{2}{|c|}{ The TG curve } & \multicolumn{2}{|c|}{ The DTG curve } \\
\cline { 2 - 6 } & $\begin{array}{c}\mathbf{T}_{\text {melting, }} \\
{ }^{\circ} \mathbf{C}\end{array}$ & $\mathbf{T}_{\mathbf{1}},{ }^{\circ} \mathbf{C}$ & $\mathbf{T}_{\mathbf{2}},{ }^{\circ} \mathbf{C}$ & $\mathbf{T}_{\mathbf{1}},{ }^{\circ} \mathbf{C}$ & $\mathbf{T}_{\mathbf{2}},{ }^{\circ} \mathbf{C}$ \\
\hline Sample \#1 & 212 & 257 & 458 & 236 & 440 \\
\hline Sample \#2 & 205 & 240 & 432 & 229 & 436 \\
\hline Sample \#3 & 210 & 234 & 448 & 288 & 458 \\
\hline Sample \#4 & 200 & 273 & 430 & 273 & 437 \\
\hline
\end{tabular}


Possibility, expediency and effectiveness of the repeated utilize of the out-of use polycaproamide screen are stipulated by physical and chemical properties of the material. In particular, molecular weight (MW) of the polymer is of great importance that affects strength and technological properties of the recycled materials and products made from them. Therefore, evaluation of the possible impact of the filter screens operating conditions on the polymer characteristic is an important stage of this study. One of the parameters depending on the MW is the greatest Newtonian viscosity $\eta_{o}$, one of the main rheological characteristics of polymer melts. These two values are associated with the exponentiation equation [5-7]:

$$
\eta_{o}=K^{1} \cdot M^{b}
$$

where $K^{l}$ - empirical constant; $M$ - molecular weight; $b$ - value of the exponent close to value 3.4 .

For a wide range of industrial polymers that differ only in molecular weight, it can be assumed with sufficient for calculations accuracy that their molecular mass distribution, which significantly affects the properties of polymers, is similar. Therefore, a change of the $\eta_{o}$ value according to equation (1) could be determined depending on the average mass, average number, or average viscosity molecular weight. For PA-6, the constant $K^{l}=$ $1.01 \cdot 10^{-13}, b=3.6$, the $\eta_{o}$ dependence is determined from the average molecular weight of $M_{n}[8,9]$, so equation (1) takes the following form:

$$
\eta_{o}=1.01 \cdot 10^{-13} M_{n}^{3.6}
$$

Thus, rheological measurements could be applied to estimate the change of the polymer MW.

One of the rheological (and, at the same time, technological) characteristics of polymers is the melt flow index $(M F I)$, which is determined on a cargo capillary viscometer as per standard methods. It was determined $[10,11]$ that the effective melt viscosity $\eta$, the maximum value of which is $\eta_{o}$, is related to the MFI by the following dependence:

$$
\eta=0.5 \frac{g \cdot \rho \cdot M_{g}}{M F I},
$$

where $\mathrm{g}$ - gravitational acceleration; $\rho$ - polymer melt density at test temperature; $M_{g}-$ weight of viscometer load; $M F I$ - melt flow index.

From the above said, it follows that the polymer possible change in MW during the product operation could be estimated by possible change in the MFI value. For all studied samples (Table 1), the MFI value was determined under the conditions regulated for the PA-6: $235^{\circ} \mathrm{C}$ temperature and $21.6 \mathrm{H}$ load on the piston. The obtained results are in Table 2 .

Table 2. Melt flow index $M F I$ and density of the samples under study

\begin{tabular}{|c|c|c|c|c|}
\hline $\begin{array}{c}\text { Characteristics of } \\
\text { the material un- } \\
\text { der study }\end{array}$ & \multicolumn{4}{|c|}{ Samples of the material under study } \\
\cline { 2 - 5 } & Sample \#1 & Sample \#2 & Sample \#3 & Sample \#4 \\
\hline $\begin{array}{c}\text { Melt flow index, } \\
\text { g/10 min }\end{array}$ & 1.21 & 1.53 & 2.25 & 1.46 \\
\hline Density, g/sm 3 & 1.133 & 1.187 & 1.149 & 1.17 \\
\hline
\end{tabular}

Due to the fact that the range of the $M F I$ values for various polyamide brands and compositions based on them, depending on conditions of the synthesis, mass fraction of extract- 
ed substances and presence of modifying additives varies widely (from 1.15 to $23.0 \mathrm{~g} / 10$ min) [12], a comparative analysis was performed relatively to the experimentally obtained values of this parameter. Samples \#\# 2 and 4 have up to $20 \%$ of coal, therefore, they can be considered as compositions with a certain amount of filler (coal), and samples \#\# 1 and 3 are not filled out.

The melt flow index of the screen that is out-of use and washed from coal is almost 2 times greater (on $86 \%$ ) than the unused screen. This could be due to the specifics of using screens in vacuum filters in constant contact with water, since polycaproamide is a hygroscopic material and has an increased water absorption. This leads to an increase in polymer moisture and a partial hydrolytic degradation process, which slightly reduces the molecular weight and reduces the viscosity of the melt. This coincides with the results given in the paper [12], where viscosity of the recycled $P A-6$ was lower than of the primary one, while the largest difference was found in the sample with increased moisture. The $M F I$ value for the out-of use screen with a coal content up to $20 \%$ (sample 2) and obtained on its basis recycled granular material (sample 4) is also higher than the unused screen on $26 \%$ and $21 \%$, respectively. A significant decrease in the viscosity level compared to sample \#3 is due to the presence of coal in the composition, which in this case plays a role of a filler. However, it does not prevent molecular weight reduction as a result of the partial destruction process during operation, but it significantly improves the technological properties of the recycled material.

It is known [2] that primary and recycled polymer raw materials differ slightly in density, which is confirmed by the research. For primary PA-6, density is regulated in the 1.13$1.15 \mathrm{~g} / \mathrm{cm}^{3}$ range, in samples without coal content (Table 2), this indicator is in the same range. Samples \#\#2 and 4 have a slightly higher density $\left(1.17-1.187 \mathrm{~g} / \mathrm{cm}^{3}\right)$ that is explained by the coal content in the material.

Technological methods to evaluate thermal stability are based on the study of phenomena occurring during degradation, changes in polymer properties such as molecular weight, melt viscosity and etc. Measurements are usually made at low speed and shear stress to avoid mechanical stress influence. Such modes are usually implemented to determine melt flow index. A specified parameter of thermal stability $T$ is the ratio of the polymer melt flow index $M F I_{t}$ after its heating up and holding for a certain time $\mathrm{t}$ (usually $\mathrm{t}=10,20$ or 30 minutes) to the polymer melt flow index MFI after its heating up during time $t_{\text {standard }}$ under standard conditions required to determine the MFI $\left(\mathrm{t}_{\text {standard }}=5\right.$ minutes $)$

$$
T=\frac{M F I_{t}}{M F I},
$$

Polymer is considered to be thermally stable in viscosity if the $M F I$ (viscosity) changes on $15-20 \%$ or less during the time $t$, i.e. the $\mathrm{T}$ value is in the range $(0.85-0.8)<\mathrm{T}<(1.15-$ $1.2)$.

Results of the thermal stability determining for the studied $\mathrm{T}$ samples are in Table 3. Melts of the unused and washed films (samples \#\#1 and 3) with an increase in the holding time at temperature are characterized by a decrease in viscosity; the period of thermal stability for them is 10 minutes. Melt viscosity of the film with coal residues (sample \#2) and melt viscosity of the recycled polycaproamide (sample \#4), on the contrary, increases, and satisfactory thermal stability is maintained up to $20 \mathrm{~min}$.

It should be noted that the obtained thermal stability values could be considered as satisfactory, since according to the literature data [13], the period of thermal stability as per change in viscosity on $15 \%$ for the primary PA-6 at a slightly higher temperature of $240^{\circ} \mathrm{C}$ is within 11-15 $\mathrm{min}$. 
The disadvantages of PA-6 include rather high level of water absorption, i.e. the amount of water absorbed by the polymer during 24 hours of being in water at temperature $23^{\circ} \mathrm{C}$, that is 2.2-2.8 \% according to [14] (according to other sources [15] is up to $3.5 \%$ ). However, it does not matter when it is used as a filter material for dewatering of coal flotation concentrates and slurries.

Table 3. Values of thermal stability of the samples under study

\begin{tabular}{|c|c|c|c|c|c|c|c|c|}
\hline \multirow{2}{*}{$\begin{array}{c}\text { Samples } \\
\text { of the } \\
\text { material } \\
\text { under } \\
\text { study }\end{array}$} & \multicolumn{6}{|c|}{ Melts holding time at temperature to determine } \\
\cline { 2 - 9 } & $\mathbf{M F I}$ & $\boldsymbol{T}$ & $\boldsymbol{M F I}_{\boldsymbol{t}}$ & $\boldsymbol{T}$ & $\boldsymbol{M F I}_{\boldsymbol{t}}$ & $\boldsymbol{T}$ & $\boldsymbol{M F I}_{\boldsymbol{t}}$ & $\boldsymbol{T}$ \\
\hline Sample\#1 & 1.21 & - & 1.32 & 1.09 & 1.51 & 125 & 1.6 & 1.32 \\
\hline Sample\#2 & 1.53 & - & 1.45 & 0.95 & 1.31 & 0.86 & 1.17 & 0.76 \\
\hline Sample\#3 & 2.25 & - & 1.97 & 0.87 & 1.78 & 0.79 & 1.72 & 0.76 \\
\hline Sample\#4 & 1.46 & - & 1.68 & 1.15 & 1.75 & 1.2 & 1.98 & 1.36 \\
\hline
\end{tabular}

In this paper, saturation by moisture kinetics in the air by unused screen (sample $\# 1$ ) during period of $0-150$ minutes at the ambient temperature $22^{\circ} \mathrm{C}$ was studied. Initial polymer moisture after pre-drying was equal to $0.18 \%$ and did not change during 0 to 30 minutes. The subsequent time of moisture saturation is divided into two distinct periods: the first one lasted from 30 to 60 minutes and was characterized by intensive saturation by moisture, while the rate of increase in material moisture was $5.67 \times 10^{-3} \%$ per minute. For PA-6 maximum regulated moisture limit of $0.2 \%$ was reached in about 50 minutes, and the polymer moisture at 60 minutes was $0.26 \%$. In the second period (from 60 to 150 minutes), the rate of moisture saturation fell exactly next order, and the final moisture content of the material reached $0.31 \%$.

\section{Conclusions}

Finally we can draw the following conclusions:

- the process of utilizing of the filter material does not lead to any serious changes in the thermal properties, density, thermal stability and saturation kinetics of the polycaproamide by moisture;

- the increase in polymer moisture due to constant contact with water during filtration contributes to a partial hydrolytic degradation process that slightly reduces the molecular weight and viscosity of the polymer melt;

- the presence of coal in the compositions under the study revealed that improving the technological properties and increasing viscosity of the melt could be carried out by introducing fillers or other modifying additives into the polymer;

- the results of the conducted research proved that the filter material after its operation is a valuable raw material, since it has the required complex of properties and is suitable for obtaining marketable recycled polycaproamide, from which a wide range of products for various purposes can be manufactured.

\section{References}

1. L. Starke, Verwerten von Plastabfallen und Plastaltstoffen (VEB Deutscher Verlag fur Grundstoffindustrie, Leipzig, 1984)

2. H. Arimoto, Polymer Sci., 2, 2283 (1964)

3. J. Dechant, H. Hoyme, Jenaer Bdsch., 10, 210 (1965) 
4. P. Bouriot, Bull. Inst., Texstile France, 11, 7 (1957)

5. A. Ya. Malkin, Av. I. Isayev, Rheology: conceptions, methods, applications (ChemTec Publishing, Toronto, Canada, 2005)

6. G. S. Berri, T. G. Fox, Adv. Polymtr Sci., 5, 261 (1968)

7. F. N. Cogswell, J. C. McGowan, Britich Polymer J., 4:3, 183 (1972)

8. T. G. Fox, P. G. Flory, Amer. Chem. Soc., 70, 2384 (1948)

9. F. N. Cogswell, Plastics and Polymers, 41:39, 151 (1973)

10. R. E. Wiley, Plast. Technol., v. 7, 5, 45 (1961)

11. R. M. McGlamery, A. A. Harban, Mater. Res. Stand., 3:12, 1003 (1963)

12. Ed.: Francesco La Mantia, Handbook of Plastics Recycling (Rapra Technology, Shrewsbury, 2002)

13. H. G. Mosle, Plast. und Kautsch., Jg. 28, 7, 409 (1981)

14. J. Brunner, Kunstoffe-Plastics, 9, 37 (1975)

15. K. V. Golman, Plastics and Polymers, 37, 309 (1969) 